Article

\title{
Unique existence of globally asymptotical input-to-state stability of positive stationary solution for impulsive Gilpin-Ayala competition model with diffusion and delayed feedback under Dirichlet zero boundary value
}

\author{
Ruofeng Rao ${ }^{1,2}$ \\ 1 Department of Mathematics, Chengdu Normal University, Chengdu 61130, China; ruofengrao@163.com or \\ ruofengrao@cdnu.edu.cn \\ 2 Institute of Financial Mathematics, Chengdu Normal University, Chengdu 61130, China
}

check for updates

\begin{abstract}
By partly generalizing the Lipschitz condition of existing results to the generalized Lipschitz one, the author utilizes a fixed point theorem, variational method and Lyapunov function method to derive the unique existence of globally asymptotical input-to-state stability of positive stationary solution for Gilpin-Ayala competition model with diffusion and delayed feedback under Dirichlet zero boundary value. Remarkably, it is the first paper to derive the unique existence of the stationary solution of reaction-diffusion Gilpin-Ayala competition model, which is globally asymptotical input-to-state stability. And numerical examples illuminate the effectiveness and feasibility of the proposed methods.
\end{abstract}

Keywords: Gilpin-Ayala competition model; globally asymptotical stability; Lyapunov function; Markovian jumping

\section{Introduction}

Delayed ecosystem or reaction-diffusion ecosystem has been investigated for a long time (see, e.g. $[1-4,10,12-14,16]$ and the references therein). But most of the related literature only involved in the Neumann zero boundary value. In real world, Dirichlet zero boundary value can sometimes better simulate the population ecology, for example, the population density of deep-sea fish at the edge of their life circle is zero, and out of the circle may mean that they cannot adapt to the environment. Besides, the delayed feedback model is introduced in this paper, for the larval individuals in the population often have a certain growth period, and only adults can participate in the food competition among populations. Such delayed feedback models are not only suitable for biological population competition model, but also common to other dynamic models ([14-16]). In addition, Markov models can always simulate the competition systems of biological population with random factors and other dynamical systems $([9,17])$. In addition, multiple-species competition models are always linear ones . For example, even in 2017, Yuanyuan Liu and Youshan Tao investigated the following the following two-species linear competition model with cross-diffusion for one species under Neumann boundary value ([4]):

$$
\left\{\begin{aligned}
\frac{\partial u}{\partial t} & =\Delta\left[\left(d_{1}+a_{12} v\right) u\right]+\mu_{1} u\left(1-u-a_{1} v\right), \quad x \in \Omega, t>0 \\
0 & =\Delta v+\mu_{2} v\left(1-v-a_{2} u\right), \quad x \in \Omega, t>0 \\
\frac{\partial u}{\partial v} & =\frac{\partial v}{\partial v}=0, \quad x \in \partial \Omega, t>0 \\
u(x, 0) & =u_{0}(x), \quad x \in \Omega .
\end{aligned}\right.
$$


Until 1973, Gilpin and Ayala found that the model did not match a series of experimental data well([5]). Via accurate data analysis, they proposed the following nonlinear competition model with two-species:

$$
\left\{\begin{array}{l}
\dot{x}_{1}(t)=x_{1}(t)\left[b_{1}-a_{11} x_{1}^{\theta_{1}}(t)-a_{12} x_{2}(t)\right], \\
\dot{x}_{2}(t)=x_{2}(t)\left[b_{2}-a_{21} x_{1}(t)-a_{22} x_{2}^{\theta_{2}}(t)\right],
\end{array}\right.
$$

in which $\theta_{1}, \theta_{2}$ represent the nonlinear density restrictions. As pointed out in [6-8] that the nonlinear density restrictions model can match well the experimental data on drosophila melanogasters when $\theta_{i}$ was far less than 1. From then, Gilpin-Ayala ecosystems have been investigated extensively (see, e.g. $[3,12,13,17])$. Even various reaction-diffusion Gilpin-Ayala competition models were investigated under Neumann boundary value (see, e.g. [2-4]). But seldom reaction-diffusion two-species competition models were studied under Dirichlet boundary value. In fact, there are many cases suitable to the Dirichlet boundary problem. For example, deep sea fish live in a certain range of three-dimensional waters, and in their area edge, the population density of deep sea fish is zero. Besides, the living range of some pollens is also affected by their regional environment. They only spread in a certain area, and the population density of the living pollens on the edge of the area is zero. Furthermore, input-to-state stability was studied in many literature involved in various dynamical systems (see [18-22] ), which is also suitable to ecosystem. In fact, putting a certain amount of food and small fry in the fish pond can be seen as the external input, which can make the dynamic of the ecosystem stabilized at a positive equilibrium point. By employing the methods used in my another paper [11], I shall utilize a fixed point theorem, variational method, and Lyapunov function method to derive the unique existence of the stationary solution of reaction-diffusion Gilpin-Ayala competition model, which is globally asymptotical input-to-state stability.

This paper involves in the following innovations or novelties:

$\star$ It is the first paper to derive the unique existence of the stationary solution of reaction-diffusion Gilpin-Ayala competition model, which is globally asymptotical input-to-state stability.

$\star$ Different from Neumann boundary problem, the non-zero constant equilibrium point is not the solution for the ecosystem with Dirichlet boundary value (see [11]), which brings about more mathematical difficulties.

$\star$ Partly generalizing the Lipschitz condition of [11, Theorem 3.1] or [11, Theorem 3.2] to the Lipschitz one in the broad sense.

Throughout of this paper, the author denotes by $I$ the identity matrix. Besides, $\left\|u_{i}\right\|=$ $\sqrt{\int_{\Omega_{\sigma}}\left|\nabla u_{i}\right|^{2} d x}$, and $\|u\|^{2}=\sum_{i=1}^{2}\left\|u_{i}\right\|^{2}$, for $u=\left(u_{1}(x), u_{2}(x)\right)^{T}$ with $u_{i} \in H_{0}^{1}(\Omega)$. Denote by $\lambda_{1}$ the first positive eigenvalue of Laplace operator $-\Delta$ in $H_{0}^{1}(\Omega)$. For vectors $u=\left(u_{1}, u_{2}\right)^{T}, v=\left(v_{1}, v_{2}\right)^{T}$, I denote $|u|=\left(\left|u_{1}\right|,\left|u_{2}\right|\right)^{T}$, and $u \leqslant v$ implies $u_{i} \leqslant v_{i}, i=1$, 2. Matrices $A<B$ means that the symmetric matrices $A, B$ satisfies $(B-A)$ is a positive definite matrix. Denote $|C|=\left(\left|c_{i j}\right|\right)_{2 \times 2}$ for matrix $C=\left(c_{i j}\right)_{2 \times 2}$.

\section{System descriptions and preparations}

Denote by $(Y, \mathcal{F}, \mathbb{P})$ the complete probability space with a natural filtration $\left\{\mathcal{F}_{t}\right\}_{t \geq 0}$. Let $S=$ $\left\{1,2, \cdots, n_{0}\right\}$ and the random form process $\{r(t):[0,+\infty) \rightarrow S\}$ be a homogeneous, finite-state Markovian process with right continuous trajectories with generator $\Pi=\left(\gamma_{i j}\right)_{n_{0} \times n_{0}}$ and transition probability from mode $i$ at time $t$ to mode $j$ at time $t+\delta, i, j \in S$,

$$
\mathbb{P}(r(t+\delta)=j \mid r(t)=i)=\left\{\begin{array}{l}
\gamma_{i j} \delta+o(\delta), \quad j \neq i \\
1+\gamma_{i j} \delta+o(\delta), \quad j=i,
\end{array}\right.
$$


where $\gamma_{i j} \geqslant 0$ is transition probability rate from $i$ to $j(j \neq i)$ and $\gamma_{i i}=-\sum_{j=1, j \neq i}^{n_{0}} \gamma_{i j}, \delta>0$ and $\lim _{\delta \rightarrow 0} o(\delta) / \delta=0$.

Consider the following delayed feedback system :

$$
\left\{\begin{array}{l}
\frac{\partial u_{1}}{\partial t}=d_{1} \Delta u_{1}+u_{1}\left(b_{1}-a_{11} u_{1}^{\theta_{1}}-a_{12} u_{2}\right)+k_{1}(r(t))\left[u_{1}-u_{1}\left(t-\tau_{1}(t), x\right)\right]+\chi_{1}, \quad t \geqslant 0, x \in \Omega, \\
\frac{\partial u_{2}}{\partial t}=d_{2} \Delta u_{2}+u_{2}\left(b_{2}-a_{21} u_{1}-a_{22} u_{2}^{\theta_{2}}\right)+k_{2}(r(t))\left[u_{2}-u_{2}\left(t-\tau_{2}(t), x\right)\right]+\chi_{2}, \quad t \geqslant 0, x \in \Omega \\
u_{1}(t, x)=u_{2}(t, x)=0, \quad t \geqslant 0, x \in \partial \Omega
\end{array}\right.
$$

where $\Omega$ is a domain in $\mathbb{R}^{3}$ with smooth boundary $\partial \Omega, \chi_{i}$ is a bounded continuous disturbance input with $\chi(x)=\left(\chi_{1}(x), \chi_{2}(x)\right)^{T}$ and $0<\left|\chi_{i}(x)\right|<J_{i}(i=1,2)$, and $k_{1}(r(t))$ and $k_{2}(r(t))$ are feedback benefit coefficients at mode $r(t)=r \in S$. Below, we denote $k_{1}(r(t))=k_{1 r}, k_{2}(r(t))=k_{2 r}$ for simple.

Remark 1. Here, we assume $\Omega \subset \mathbb{R}^{3}$. And if two species live in two dimensional plane, we can assume $u_{i}(t, x)=u_{i}\left(t, x_{1}, x_{2}, x_{3}\right)=u_{i}\left(t, x_{1}, x_{2}, \cdot\right)$, independent of the third dimension, where $x=$ $\left(x_{1}, x_{2}, x_{3}\right)^{T} \in \Omega$.

Assume that $\left(u_{1}^{*}(x), u_{2}^{*}(x)\right)$ is a positive stationary solution of the system (2.1). Set

$$
\left\{\begin{array}{c}
U_{1}=u_{1}-u_{1}^{*}(x) \\
U_{2}=u_{2}-u_{2}^{*}(x)
\end{array}\right.
$$

and the stationary solution $\left(u_{1}^{*}(x), u_{2}^{*}(x)\right)$ of the system $(2.1)$ corresponds to the zero solution $(0,0)$ of the following system:

$$
\begin{cases}\frac{\partial U_{1}}{\partial t}=d_{1} \Delta U_{1}+b_{1} U_{1}-\Phi_{1}\left(U_{1}, U_{2}\right)+k_{1 r}\left[U_{1}-U_{1}\left(t-\tau_{1}(t), x\right)\right], & t \geqslant 0, x \in \Omega \\ \frac{\partial U_{2}}{\partial t}=d_{2} \Delta U_{2}+b_{2} U_{2}-\Phi_{2}\left(U_{1}, U_{2}\right)+k_{2 r}\left[U_{2}-U_{2}\left(t-\tau_{2}(t), x\right)\right], & t \geqslant 0, x \in \Omega \\ U_{1}(t, x)=U_{2}(t, x)=0, \quad t \geqslant 0, x \in \partial \Omega & \end{cases}
$$

or

$$
\begin{cases}\frac{\partial U_{1}}{\partial t}=d_{1} \Delta U_{1}+\left(b_{1}+k_{1 r}\right) U_{1}-\Phi_{1}\left(U_{1}, U_{2}\right)-k_{1 r} U_{1}\left(t-\tau_{1}(t), x\right), \quad t \geqslant 0, x \in \Omega, \\ \left.\frac{\partial U_{2}}{\partial t}=d_{2} \Delta U_{2}+\left(b_{2}+k_{2 r}\right) U_{2}-\Phi_{2}\left(U_{1}, U_{2}\right)-k_{2 r} U_{2}\left(t-\tau_{2}(t), x\right)\right], \quad t \geqslant 0, x \in \Omega, \\ U_{1}(t, x)=U_{2}(t, x)=0, \quad t \geqslant 0, x \in \partial \Omega,\end{cases}
$$

where we denote $U=\left(U_{1}, U_{2}\right)^{T}$, and

$$
\begin{aligned}
& \Phi_{1}(U)=\left(U_{1}+u_{1}^{*}(x)\right)\left[a_{11}\left(U_{1}+u_{1}^{*}(x)\right)^{\theta_{1}}+a_{12}\left(U_{2}+u_{2}^{*}(x)\right)\right]-u_{1}^{*}(x)\left(a_{11} u_{1}^{*}(x)^{\theta_{1}}+a_{12} u_{2}^{*}(x)\right), \\
& \Phi_{2}(U)=\left(U_{2}+u_{2}^{*}(x)\right)\left[a_{21}\left(U_{1}+u_{1}^{*}(x)\right)+a_{22}\left(U_{2}+u_{2}^{*}(x)\right)^{\theta_{2}}\right]-u_{2}^{*}(x)\left(a_{21} u_{1}^{*}(x)+a_{22} u_{2}^{*}(x)^{\theta_{2}}\right) .
\end{aligned}
$$

The following system is the system (2.3) in form of vector-matrix:

$$
\left\{\begin{array}{l}
\frac{\partial U}{\partial t}=D \Delta U+\left(B+K_{r}\right) U-\Phi(U)-K_{r} U(t-\tau(t), x), \quad t \geqslant 0, x \in \Omega, \\
U(t, x)=0, \quad t \geqslant 0, x \in \partial \Omega
\end{array}\right.
$$

where $U=\left(U_{1}, U_{2}\right)^{T}, U(t-\tau(t), x)=\left(U\left(t-\tau_{1}(t), x\right), U\left(t-\tau_{2}(t), x\right)\right)^{T}, \Phi(U)=\left(\Phi_{1}(U), \Phi_{2}(U)\right)^{T}$ and

$$
D=\left(\begin{array}{cc}
d_{1} & 0 \\
0 & d_{2}
\end{array}\right), A_{k}=\left(\begin{array}{cc}
a_{1}^{(k)} & 0 \\
0 & a_{2}^{(k)}
\end{array}\right), B=\left(\begin{array}{cc}
b_{1} & 0 \\
0 & b_{2}
\end{array}\right), K_{r}=\left(\begin{array}{cc}
k_{1 r} & 0 \\
0 & k_{2 r}
\end{array}\right) .
$$


Under impulse control on (2.5), one can get the following system

$$
\left\{\begin{array}{l}
\frac{\partial U}{\partial t}=D \Delta U+\left(B+K_{r}\right) U-\Phi(U)-K_{r} U(t-\tau(t), x), \quad t \geqslant 0, t \neq t_{k}, x \in \Omega \\
U\left(t_{k}^{+}, x\right)=A_{k} U\left(t_{k}^{-}, x\right), \quad k=1,2 \ldots \\
U(t, x)=0, \quad t \geqslant 0, x \in \partial \Omega
\end{array}\right.
$$

where $U\left(t_{k}^{-}, x\right)=U\left(t_{k}, x\right)$ for all $i=1,2, k=1,2, \cdots$. Besides, the bounded initial value of the system (2.7) is proposed as follows,

$$
U_{1}(s, x)=\phi_{1}(s, x) \geqslant 0, U_{2}(s, x)=\phi_{2}(s, x) \geqslant 0, \quad s \in[-\tau, 0], x \in \Omega,
$$

or

$$
U(s, x)=\phi(s, x) \geqslant 0, \quad s \in[-\tau, 0], x \in \Omega,
$$

where $\phi(s, x)=\left(\phi_{1}(s, x), \phi_{2}(s, x)\right)^{T}$.

\section{Unique existence of globally asymptotically stable positive stationary solution}

Firstly assume that $\theta_{i} \in(0,1)$ for $i=1,2$, just like [6-8].

Next, the following assumption on the population density may be necessary:

(H1) There are positive numbers $M_{i}, N_{i}$ such that

$$
0<N_{1} \leqslant u_{1} \leqslant M_{1}, \quad 0<N_{2} \leqslant u_{2} \leqslant M_{2}
$$

Remark 2. Everyone knows the fact that the population density of any species must have the bounded below, or the species will die out. For example, When the population density of whales is lower than a certain degree, it will be difficult for male and female whales to meet each other in the vast sea, leading to the extinction of the species. Besides, due to the limited resource, the population density of any species must have an supper boundedness.

Next, the following existence of positive stationary solution comes mainly from [11, Theorem 3.1]. Of course, the ecosystem (2.5) is involved in non-Lipschitz functions, and so the author has to generalize the first conclusion of [11, Theorem 3.1] from the Lipschitz condition to the generalized Lipschitz condition.

Theorem 3.1. Suppose the condition (H1) holds, $\theta_{i} \in(0,1)$ for $i=1,2$ and $0<\left|\chi_{i}\right|<J_{i}$ with $J=\left(J_{1}, J_{2}\right)^{T}$,

$$
0 \leqslant g\left(u^{*}(x)\right)-J \leqslant g\left(u^{*}(x)\right)+J \leqslant c D E
$$

where $g(u)=\left(g_{1}\left(u_{1}, u_{2}\right), g_{2}\left(u_{1}, u_{2}\right)\right)^{T}$, and

$$
g_{1}\left(u_{1}, u_{2}\right)=u_{1}\left(b_{1}-a_{11} u_{1}^{\theta_{1}}-a_{12} u_{2}\right), \quad g_{2}\left(u_{1}, u_{2}\right)=u_{2}\left(b_{2}-a_{21} u_{1}-a_{22} u_{2}^{\theta_{2}}\right),
$$

then the system (2.1) possesses at least one positive bounded stationary solution $\left(u_{1}^{*}, u_{2}^{*}\right)$.

Proof. Firstly definite the so-called generalized Lipschitz condition as follows,

$f\left(u_{1}, u_{2}\right)$ is said to satisfy the generalized Lipschitz condition if there are constants $\bar{l}_{1}, \bar{l}_{2}>0$ such that

$$
\left|f\left(u_{1}, u_{2}\right)-f\left(v_{1}, v_{2}\right)\right| \leqslant \bar{l}_{1}\left|u_{1}-v_{1}\right|+\bar{l}_{2}\left|u_{2}-v_{2}\right|, \quad u_{i}, v_{i} \in \mathbb{R}^{1} .
$$

In fact, the first conclusion of [11, Theorem 3.1] holds still if the Lipschitz conditions are replaced with the generalized Lipschitz condition. And hence, Theorem 3.1 is the direct corollary of [11, Theorem 3.1]. However, in view of the integrity of the proof, the author is willing to prove it in details. 
Indeed, let $\left(u_{1}(x), u_{2}(x)\right)$ is the stationary solution, satisfying

$$
\begin{cases}d_{1} \Delta u_{1}+g_{1}\left(u_{1}, u_{2}\right)+\chi_{1}=0, & x \in \Omega, \\ d_{2} \Delta u_{2}+g_{2}\left(u_{1}, u_{2}\right)+\chi_{2}=0, & x \in \Omega, \\ u_{1}(x)=u_{2}(x)=0, \quad x \in \partial \Omega, & \end{cases}
$$

The condition (H1) yields that there are four positive constants $l_{1}, l_{2}, l_{3}$ and $l_{4}$ such that

$$
\left|g_{1}\left(u_{1}, u_{2}\right)-g_{1}\left(v_{1}, v_{2}\right)\right| \leqslant l_{1}\left|u_{1}-v_{1}\right|+l_{2}\left|u_{2}-v_{2}\right|, \quad u_{i}, v_{i} \in \mathbb{R}^{1}
$$

and

$$
\left|g_{2}\left(u_{1}, u_{2}\right)-g_{2}\left(v_{1}, v_{2}\right)\right| \leqslant l_{3}\left|u_{1}-v_{1}\right|+l_{4}\left|u_{2}-v_{2}\right|, \quad u_{i}, v_{i} \in \mathbb{R}^{1}
$$

where

$l_{1}=b_{1}+a_{11}\left(1+\theta_{1}\right) M_{1}^{\theta_{1}}+a_{12} M_{2}, \quad l_{2}=a_{12} M_{1}, \quad l_{3}=a_{21} M_{2}, \quad l_{4}=b_{2}+a_{22}\left(1+\theta_{2}\right) M_{2}^{\theta_{2}}+a_{21} M_{1}$.

In fact, $0<\theta_{i}<1$ and $(\mathrm{H} 1)$ yield

$$
\begin{aligned}
\left|g_{1}\left(u_{1}, u_{2}\right)-g_{1}\left(v_{1}, v_{2}\right)\right| & =\left|\left[u_{1}\left(b_{1}-a_{11} u_{1}^{\theta_{1}}-a_{12} u_{2}\right)\right]-\left[v_{1}\left(b_{1}-a_{11} v_{1}^{\theta_{1}}-a_{12} v_{2}\right)\right]\right| \\
& \leqslant\left[b_{1}+a_{11}\left(1+\theta_{1}\right) M_{1}^{\theta_{1}}+a_{12} M_{2}\right]\left|u_{1}-v_{1}\right|+a_{12} M_{1}\left|u_{2}-v_{2}\right|, \\
\left|g_{2}\left(u_{1}, u_{2}\right)-g_{2}\left(v_{1}, v_{2}\right)\right| & =\left|\left[u_{2}\left(b_{2}-a_{21} u_{1}-a_{22} u_{2}^{\theta_{2}}\right)\right]-\left[v_{2}\left(b_{2}-a_{21} v_{1}-a_{22} v_{2}^{\theta_{2}}\right)\right]\right| \\
& \leqslant a_{21} M_{2}\left|u_{1}-v_{1}\right|+\left[b_{2}+a_{22}\left(1+\theta_{2}\right) M_{2}^{\theta_{2}}+a_{21} M_{1}\right]\left|u_{2}-v_{2}\right|,
\end{aligned}
$$

which derives (3.8).

If the stationary solution of the system (2.1) exists, I may denote it by $u^{*}(x)=\left(u_{1}^{*}(x), u_{2}^{*}(x)\right)^{T}$.

Define the operator $\mathfrak{M}:\left[C\left(\overline{\Omega_{\sigma}}\right)\right]^{2} \rightarrow\left[C\left(\overline{\Omega_{\sigma}}\right)\right]^{2}$ as follows,

$$
\mathfrak{M}=\left(\begin{array}{cc}
-\Delta & 0 \\
0 & -\Delta
\end{array}\right) \text {. }
$$

The operator $\mathfrak{M}$ has the inverse operator $\mathfrak{M}^{-1}$ as follows,

$$
\mathfrak{M}^{-1}=\left(\begin{array}{cc}
(-\Delta)^{-1} & 0 \\
0 & (-\Delta)^{-1}
\end{array}\right)
$$

where $\mathfrak{M}^{-1}:\left[C\left(\overline{\Omega_{\sigma}}\right)\right]^{2} \rightarrow\left[C\left(\overline{\Omega_{\sigma}}\right)\right]^{2}$ is a linear compact positive operator (see, e.g. [11]), and

$$
\left\{\begin{array}{l}
\mathfrak{M} u^{*}(x)=D^{-1} g\left(u^{*}(x)\right)+D^{-1} \chi, \quad x \in \Omega \\
u^{*}(x)=0, x \in \partial \Omega
\end{array}\right.
$$

It is obvious that $\left(D^{-1} g\left(u^{*}(x)\right)+D^{-1} \chi\right)$ is continuous for all the variables $x, u_{1}^{*}, u_{2}^{*}$. Define

$$
\mathfrak{K}=\left\{\varphi(x) \in\left[C\left(\overline{\Omega_{\sigma}}\right)\right]^{2}: \varphi(x) \geqslant 0, x \in \Omega ; \varphi(x)=0, x \in \partial \Omega\right\},
$$

then $\mathfrak{K}$ is a positive cone, which must be a closed convex subset of $\left[C\left(\overline{\Omega_{\sigma}}\right)\right]^{2}$. Define an operator $\mathfrak{T}: \mathfrak{K} \rightarrow \mathfrak{K}$ such that

$$
\mathfrak{T} \varphi=\mathfrak{M}^{-1}\left(D^{-1} g\left(u^{*}(x)\right)+D^{-1} \chi\right), \quad \varphi \in \mathfrak{K} .
$$


Because $\mathfrak{M}^{-1}$ is the linear positive compact operator, and $\left(D^{-1} g\left(u^{*}(x)\right)+D^{-1} \chi\right)$ is positive continuous, one can conclude that $\mathfrak{T}: \mathfrak{K} \rightarrow \mathfrak{K}$ is a positive compact operator.

Next, completely similar as the proof of [11, Theorem 3.1], one can utilize the fixed point theorem ([11, Lemma 2.1]) to prove that $\mathfrak{T}$ satisfies all the assumption conditions of [11, Lemma 2.1], which implies that $\mathfrak{T}$ has at least a fixed point in $\mathfrak{K}$. And $u^{*}$ is a bounded positive solution of the system (2.1).

Next, [11, Theorem 3.2] proposes the methods which may be helpful to conclude the following uniqueness result:

Theorem 3.2. Based on the assumptions of Theorem 3.1, and suppose, in addition, the following condition is satisfied,

(H2) for any mode $r(t)=r$, there exists a scalar $\varepsilon>0$ such that

$$
\left(\begin{array}{cc}
l_{1}+\varepsilon \frac{l_{2}+l_{3}}{2} & 0 \\
0 & l_{4}+\varepsilon^{-1 \frac{l_{2}+l_{3}}{2}}
\end{array}\right)<\lambda_{1} D,
$$

then the system (2.1) possesses the unique positive bounded stationary solution $u^{*}(x)$ for $x \in \Omega_{\sigma}$ with $\left.u^{*}\right|_{\partial \Omega_{\sigma}}=0$, where $u^{*}(x)$ is the positive bounded solution in Theorem 3.1, and $l_{i}$ is defined in (3.8).

Proof. Assume both $u(x)$ and $v(x)$ are the stationary solutions of the system (2.1). Then we claim $u(x)=v(x)$.

In fact,

$$
\begin{aligned}
& (u(x)-v(x))^{T}(g(u(x))-g(v(x))) \leqslant|u-v|^{T}|g(u)-g(v)| \\
\leqslant & l_{1}\left|u_{1}-v_{1}\right|^{2}+l_{2}\left|u_{1}-v_{1}\right| \cdot\left|u_{2}-v_{2}\right|+l_{3}\left|u_{1}-v_{1}\right| \cdot\left|u_{2}-v_{2}\right|+l_{4}\left|u_{2}-v_{2}\right|^{2} \\
\leqslant & |u-v|^{T}\left(\begin{array}{cc}
l_{1}+\frac{\varepsilon_{2}+l_{3}}{2} & 0 \\
0 & l_{4}+\varepsilon^{-1 \frac{l_{2}+l_{3}}{2}}
\end{array}\right)|u-v|
\end{aligned}
$$

Below, I shall employ some methods similar as those of the proof of [11, Theorem 3.2]. Since both $u(x)$ and $v(x)$ are the stationary solutions of the system (2.1), one can see it from (3.10), variational method and the Poincare inequality that

$$
\begin{aligned}
& \lambda_{1} \int_{\Omega}|u(x)-v(x)|^{T} D|u(x)-v(x)| d x \leqslant \int_{\Omega}|\nabla(u(x)-v(x))|^{T} D|\nabla(u(x)-v(x))| d x \\
= & \int_{\Omega}(u-v)^{T}[g(u)-g(v)] d x \\
\leqslant & |u-v|^{T}\left(\begin{array}{cc}
l_{1}+\varepsilon \frac{l_{2}+l_{3}}{2} & 0 \\
0 & l_{4}+\varepsilon^{-1} \frac{l_{2}+l_{3}}{2}
\end{array}\right)|u-v| .
\end{aligned}
$$

Now the condition (H2) yields the claim via the proof by contradiction. And so the system (2.1) possesses a unique positive bounded stationaru solution $u^{*}(x)$ for $x \in \Omega_{\sigma}$ with $\left.u^{*}\right|_{\partial \Omega_{\sigma}}=0$.

Below, I shall prove that the above-mentioned positive bounded vector function $u^{*}(x)$ is globally exponentially stable, which is the unique stationary solution of the system (2.1), corresponding to the null solution of the system (2.7).

Theorem 3.3. Suppose the conditions (H1),(H2) and (3.2) hold. In addition, there is a sequence positive definite matrices $P_{r}(r \in S)$, positive numbers $w_{r}, \pi_{r}(r \in S), \varepsilon, \varepsilon_{1}, \varepsilon_{2}, \gamma, \varsigma, \lambda$ such that

$$
0<\lambda_{\max } A_{k}^{T} A_{k}<e^{-(\varsigma+\lambda)\left(t_{k+1}-t_{k}\right)}, k \in \mathbb{Z}^{+},
$$




$$
\begin{gathered}
\frac{1}{w_{r}} \lambda_{\max }\left(-2 \lambda_{1} D P_{r}+2\left(B+K_{r}\right) P_{r}+\sum_{j \in S} \gamma_{r j} P_{j}+\varepsilon_{1} P_{r} K_{r}+\varepsilon_{2} P_{r}+\varepsilon_{2}^{-1} \pi_{r} L_{\Phi}\right)+\frac{\gamma e^{\lambda \tau}}{w_{r}} \lambda_{\max }\left(\varepsilon_{1}^{-1} P_{r} K_{r}\right) \leqslant \varsigma-\lambda \\
0<w_{r} I \leqslant P_{r} \leqslant \pi_{r} I, \forall r \in S
\end{gathered}
$$

where $\gamma \geqslant \frac{1}{\lambda_{\max } A_{k}^{T} A_{k}}, k \in \mathbb{Z}^{+}$, and

$$
L_{\Phi}=2\left(\begin{array}{cc}
{\left[a_{11}\left(1+\theta_{1}\right) M_{1}^{\theta_{1}}+a_{12} M_{2}\right]^{2}+a_{21}^{2} M_{2}^{2}} & 0 \\
0 & {\left[a_{22}\left(1+\theta_{2}\right) M_{2}^{\theta_{2}}+a_{21} M_{1}\right]^{2}+a_{12}^{2} M_{1}^{2}}
\end{array}\right)
$$

then the unique positive bounded stationary solution $u^{*}(x)$ is globally exponential input-to-state stability for $0<|\chi|<J$. At the same time, the null solution of the impulsive system (2.7) with initial value (2.8) is globally exponential input-to-state stability with the convergence rate $\frac{\lambda}{2}$.

Proof. Consider the following Lyapunov function:

$$
V(t, r)=\int_{\Omega} U^{T}(t, x) P_{r} U(t, x) d x, \quad \forall r(t)=r \in S .
$$

Below, the Poincare inequality is employed to deal with the diffusion item, just like the related literature (see, e.g. [23]). Let $\mathcal{L}$ be the weak infinitesimal operator (see, e.g. [23]) such that

$$
\begin{aligned}
\mathcal{L} V(t, r) \leqslant & \int_{\Omega}\left(U^{T}\left[-2 \lambda_{1} D P_{r}+2\left(B+K_{r}\right) P_{r}+\sum_{j \in S} \gamma_{r j} P_{j}\right] U+\left[|U|^{T} P_{r}|\Phi(U)|+|\Phi(U)|^{T} P_{r}|U|\right]\right. \\
& \left.+\left[|U|^{T} P_{r} K_{r}|U(t-\tau(t), x)|+|U(t-\tau(t), x)|^{T} K_{r} P_{r}|U|\right]\right) d x, \quad t \geqslant 0, t \neq t_{k}
\end{aligned}
$$

On the other hand,

$|U|{ }^{T} P_{r} K_{r}|U(t-\tau(t), x)|+|U(t-\tau(t), x)|{ }^{T} K_{r} P_{r}|U| \leqslant \varepsilon_{1} U^{T} P_{r} K_{r} U+\varepsilon_{1}^{-1} U^{T}(t-\tau(t), x) P_{r} K_{r} U(t-\tau(t), x)$,

and

$$
|U|^{T} P_{r}|\Phi(U)|+|\Phi(U)|^{T} P_{r}|U| \leqslant \varepsilon_{2} U^{T} P_{r} U+\varepsilon_{2}^{-1} \Phi^{T}(U) P_{r} \Phi(U) \leqslant \varepsilon_{2} U^{T} P_{r} U+\varepsilon_{2}^{-1} \pi_{r} \Phi^{T}(U) \Phi(U) .
$$

Besides,

$$
\begin{aligned}
\left|\Phi_{1}(U)\right| & =u_{1}\left(a_{11} u_{1}^{\theta_{1}}+a_{12} u_{2}\right)-u_{1}^{*}(x)\left(a_{11} u_{1}^{*}(x)^{\theta_{1}}+a_{12} u_{2}^{*}(x)\right) \\
& \leqslant\left[a_{11}\left(1+\theta_{1}\right) M_{1}^{\theta_{1}}+a_{12} M_{2}\right]\left|U_{1}\right|+a_{12} M_{1}\left|U_{2}\right|
\end{aligned}
$$

Similarly,

$$
\left|\Phi_{2}(U)\right| \leqslant a_{21} M_{2}\left|U_{1}\right|+\left[a_{22}\left(1+\theta_{2}\right) M_{2}^{\theta_{2}}+a_{21} M_{1}\right]\left|U_{2}\right|
$$

In addition,

$$
\begin{aligned}
& \Phi^{T}(U) \Phi(U) \\
\leqslant & \left(\left[a_{11}\left(1+\theta_{1}\right) M_{1}^{\theta_{1}}+a_{12} M_{2}\right]\left|U_{1}\right|+a_{12} M_{1}\left|U_{2}\right|\right)^{2}+\left(a_{21} M_{2}\left|U_{1}\right|+\left[a_{22}\left(1+\theta_{2}\right) M_{2}^{\theta_{2}}+a_{21} M_{1}\right]\left|U_{2}\right|\right)^{2} \\
\leqslant & U^{T} L_{\Phi} U .
\end{aligned}
$$

It follows by (3.16)-(3.19) that 


$$
\begin{aligned}
\mathcal{L} V(t, r) \leqslant & \int_{\Omega}\left(U^{T}\left[-2 \lambda_{1} D P_{r}+2\left(B+K_{r}\right) P_{r}+\sum_{j \in S} \gamma_{r j} P_{j}\right] U+\left[|U|^{T} P_{r}|\Phi(U)|+|\Phi(U)|^{T} P_{r}|U|\right]\right. \\
& \left.+\left[|U|^{T} P_{r} K_{r}|U(t-\tau(t), x)|+|U(t-\tau(t), x)|^{T} K_{r} P_{r}|U|\right]\right) d x \\
\leqslant & \int_{\Omega} U^{T}\left[-2 \lambda_{1} D P_{r}+2\left(B+K_{r}\right) P_{r}+\sum_{j \in S} \gamma_{r j} P_{j}+\varepsilon_{1} P_{r} K_{r}+\varepsilon_{2} P_{r}+\varepsilon_{2}^{-1} \pi_{r} L_{\Phi}\right] U d x \\
& +\varepsilon_{1}^{-1} \int_{\Omega} U^{T}(t-\tau(t), x) P_{r} K_{r} U(t-\tau(t), x) d x, \quad t \geqslant 0, t \neq t_{k},
\end{aligned}
$$

which implies that for a small enough positive number $\epsilon$,

$$
\mathbb{E} V(t+\epsilon)-\mathbb{E} V(t)=\int_{t}^{t+\epsilon} \mathbb{E} \mathcal{L} V(s) d s,
$$

and letting $\epsilon \rightarrow 0$, it leads to

$$
\begin{aligned}
& D^{+} \mathbb{E} V(t, r) \\
\leqslant & \mathbb{E} \int_{\Omega} U^{T}\left[-2 \lambda_{1} D P_{r}+2\left(B+K_{r}\right) P_{r}+\sum_{j \in S} \gamma_{r j} P_{j}+\varepsilon_{1} P_{r} K_{r}+\varepsilon_{2} P_{r}+\varepsilon_{2}^{-1} \pi_{r} L_{\Phi}\right] U d x \\
& +\varepsilon_{1}^{-1} \mathbb{E} \int_{\Omega} U^{T}(t-\tau(t), x) P_{r} K_{r} U(t-\tau(t), x) d x \\
\leqslant & \frac{1}{w_{r}} \lambda_{\max }\left[-2 \lambda_{1} D P_{r}+2\left(B+K_{r}\right) P_{r}+\sum_{j \in S} \gamma_{r j} P_{j}+\varepsilon_{1} P_{r} K_{r}+\varepsilon_{2} P_{r}+\varepsilon_{2}^{-1} \pi_{r} L_{\Phi}\right] \mathbb{E} V(t, r) \\
& +\frac{1}{w_{r}} \lambda_{\max }\left(\varepsilon_{1}^{-1} P_{r} K_{r}\right) \mathbb{E} V(t-\tau(t), r)
\end{aligned}
$$

Due to the conditions (3.12)-(3.14) and the proof of [15, Theorem 3.3], we can similarly prove and obtain the following inequality:

$$
\mathbb{E} V(t, r) \leqslant M\left(\mathbb{E} \sup _{s \in[-\tau, 0]} V(s, r)\right) e^{-\lambda\left(t-t_{0}\right)}, \quad t \in\left[t_{k-1}, t_{k}\right), k \in \mathbb{Z}^{+}, r \in S .
$$

where $M>1$ is a constant.

Moreover, it follows by (3.22) and (3.14) that

$$
\left(\min _{r \in S} w_{r}\right) \mathbb{E}\|U(t)\|_{L^{2}(\Omega)}^{2} \leqslant\left(\max _{r \in S} \pi_{r}\right) M \mathbb{E}\|\phi(s)\|_{\tau}^{2} e^{-\lambda\left(t-t_{0}\right)}, \quad t \in\left[t_{k-1}, t_{k}\right), k \in \mathbb{Z}^{+},
$$

where $\|\phi(s)\|_{\tau}^{2}=\sup _{s \in[-\tau, 0]} \int_{\Omega}|\phi(s, x)|^{2} d x$. Similarly as the proof of [16, Theorem 2], one can conclude from (3.23) that

$$
\mathbb{E}\|U(t)\|_{L^{2}(\Omega)}^{2} \leqslant \frac{\max _{r \in S} \pi_{r}}{\min _{r \in S} w_{r}} M \mathbb{E}\|\phi(s)\|_{\tau}^{2} e^{-\lambda\left(t-t_{0}\right)}, \quad t \in\left[t_{k-1}, t_{k}\right], k \in \mathbb{Z}^{+},
$$

in which the positive constant $\frac{\max _{r \in S} \pi_{r}}{\min _{r \in S} w_{r}} M$ is independent of any $r \in S$. Therefore, the unique positive bounded stationary solution $u^{*}(x)$ is globally exponential input-to-state stability for $0<|\chi|<J$. At the same time, the null solution of the impulsive system (2.7) with initial value (2.8) is globally exponential input-to-state stability with the convergence rate $\frac{\lambda}{2}$. 


\section{Numerical example}

Example 4.1. Consider the following system:

$$
\left\{\begin{array}{l}
\frac{\partial u_{1}}{\partial t}=d_{1} \Delta u_{1}+u_{1}\left(b_{1}-a_{11} u_{1}^{\frac{1}{3}}-a_{12} u_{2}\right)+k_{1}(r(t))\left[u_{1}-u_{1}\left(t-\tau_{1}(t), x\right)\right]+\chi_{1}, \quad t \geqslant 0, x \in \Omega, \\
\frac{\partial u_{2}}{\partial t}=d_{2} \Delta u_{2}+u_{2}\left(b_{2}-a_{21} u_{1}-a_{22} u_{2}^{\frac{1}{5}}\right)+k_{2}(r(t))\left[u_{2}-u_{2}\left(t-\tau_{2}(t), x\right)\right]+\chi_{2}, \quad t \geqslant 0, x \in \Omega, \\
u_{1}(t, x)=u_{2}(t, x)=0, \quad t \geqslant 0, x \in \partial \Omega,
\end{array}\right.
$$

where $\theta_{1}=\frac{1}{3}, \theta_{2}=\frac{1}{5}, \Omega=\left(-\frac{1}{2}, \frac{1}{2}\right) \times\left(-\frac{1}{2}, \frac{1}{2}\right) \times\left(-\frac{1}{2}, \frac{1}{2}\right)$, then $\lambda_{1} \geqslant 3$ (see [11, Remark 14]). Let $d_{1}=0.5, b_{1}=1.1, d_{2}=0.4, b_{2}=1, J_{1}=0.003=J_{2}, \varepsilon=\varepsilon_{1}=\varepsilon_{2}=1, a_{11}=0.002, a_{12}=0.001, a_{21}=$ $0.001, a_{22}=0.002, \tau=0.01$. And $M_{1}=1.5, M_{2}=1.6, N_{1}=0.5, N_{2}=0.6$.and $c=100000$, then direct computation yields

$$
0 \leqslant g\left(u^{*}(x)\right)-J \leqslant g\left(u^{*}(x)\right)+J \leqslant c D E
$$

and

$$
\left(\begin{array}{cc}
l_{1}+\varepsilon \frac{l_{2}+l_{3}}{2} & 0 \\
0 & l_{4}+\varepsilon^{-1 \frac{l_{2}+l_{3}}{2}}
\end{array}\right)<\lambda_{1} D,
$$

which means both the conditions (3.2) and (3.9) hold.

Furthermore, let $S=\{1,2\}$, and

$$
\begin{gathered}
\Pi=\left(\gamma_{i j}\right)_{2 \times 2}=\left(\begin{array}{cc}
-0.1 & 0.1 \\
0.15 & -0.15
\end{array}\right), \quad K_{1}=\left(\begin{array}{cc}
0.0013 & 0 \\
0 & 0.0023
\end{array}\right), \quad K_{2}=\left(\begin{array}{cc}
0.0012 & 0 \\
0 & 0.0021
\end{array}\right) . \\
P_{1}=\left(\begin{array}{cc}
0.9813 & 0 \\
0 & 1.0033
\end{array}\right), \quad P_{2}=\left(\begin{array}{cc}
1.0339 & 0 \\
0 & 0.9963
\end{array}\right),
\end{gathered}
$$

and $w_{1}=0.98, \pi_{1}=1.005, w_{2}=0.99, \pi_{2}=1.07$, then the condition (3.14) holds obviously.

Assume the pulse interval $\left(t_{k+1}-t_{k}\right)=0.5$, for all $k \in \mathbb{Z}^{+}$, and

$$
A_{k} \equiv\left(\begin{array}{cc}
0.1 & 0 \\
0 & 0.2
\end{array}\right), \quad k \in \mathbb{Z}^{+}
$$

Now, we set $\gamma=26$, then we get $\gamma=26 \geqslant 25=\frac{1}{\lambda_{\max } A_{k}^{T} A_{k}}, k \in \mathbb{Z}^{+}$. Set $\varsigma=5$ and $\lambda=1$, then the direct calculation makes the condition (3.13) hold. Besides,

$$
0<\lambda_{\max } A_{k}^{T} A_{k}=0.04<0.0498=e^{-(\varsigma+\lambda)\left(t_{k+1}-t_{k}\right)}, k \in \mathbb{Z}^{+},
$$

which makes the condition (3.12) holden.

Now, all the conditions of Theorem 3.3 are satisfied. According to Theorem 3.3, the system (4.1) possesses the unique positive bounded stationary solution $u^{*}(x)$, which is globally exponential input-to-state stability with the convergence rate $\frac{\lambda}{2}=0.5$.

Example 4.2. In Example 4.1, replace the impulse quantity (4.2) with the following stronger pulse amplitude:

$$
A_{k} \equiv\left(\begin{array}{cc}
0.01 & 0 \\
0 & 0.02
\end{array}\right), \quad k \in \mathbb{Z}^{+},
$$

and the pulse interval $\left(t_{k+1}-t_{k}\right) \equiv 0.5$ remains unchanged, then we set $\gamma=630$, and hence $\gamma=630 \geqslant$ $625=\frac{1}{\lambda_{\max } A_{k}^{T} A_{k}}, k \in \mathbb{Z}^{+}$.

Set $\varsigma=10$ and $\lambda=2$, then the direct calculation makes the condition (3.15) hold. Further, 


$$
0<\lambda_{\max } A_{k}^{T} A_{k}=0.0016<0.0025=e^{-(\varsigma+\lambda)\left(t_{k+1}-t_{k}\right)}, k \in \mathbb{Z}^{+},
$$

which makes the condition (3.12) hold. Now, all the conditions of Theorem 3.3 are satisfied. According to Theorem 3.3, the system (4.1) possesses the unique positive stationary solution $\left(u_{1}^{*}, u_{2}^{*}\right)$, which is globally exponentially stabilized under impulse control with the convergence rate $\frac{\lambda}{2}=1$.

Table 1.Comparisons the influences on the convergence rate $\frac{\lambda}{2}$ under different pulse amplitude with the same other data

\begin{tabular}{|c|c|c|}
\hline & Example 4.1 & Example 4.2 \\
\hline Pulse amplitude & $\lambda_{\max } A_{k}=0.2$ & $\lambda_{\max } A_{k}=0.02$ \\
\hline Pulse intensity & smaller & bigger \\
\hline Pulse interval & $\left(t_{k+1}-t_{k}\right) \equiv 0.5$ & $\left(t_{k+1}-t_{k}\right) \equiv 0.5$ \\
\hline Pulse frequency & same & same \\
\hline Convergence rate & $\frac{\lambda}{2}=0.5$ & $\frac{\lambda}{2}=1$ \\
\hline
\end{tabular}

Remark 3. Table 1 illuminates that under the same pulse frequency, the higher the pulse intensity, the faster the convergence speed.

Example 4.3. In Example 4.1, we replace the pulse interval with $\left(t_{k+1}-t_{k}\right) \equiv 0.3$, and pulse amplitude (4.2) remains unchanged.

Now, we set $\gamma=26$, then we get $\gamma=26 \geqslant 25=\frac{1}{\lambda_{\max } A_{k}^{T} A_{k}}, k \in \mathbb{Z}^{+}$. Set $\varsigma=5.5$ and $\lambda=1.5$, then the direct calculation makes the condition (3.15) hold. Further,

$$
0<\lambda_{\max } A_{k}^{T} A_{k}=0.04<0.1225=e^{-(\varsigma+\lambda)\left(t_{k+1}-t_{k}\right)}, k \in \mathbb{Z}^{+},
$$

which makes the condition (3.12) hold.

Now, all the conditions of Theorem 3.3 are satisfied. According to Theorem 3.2, the system (4.1) possesses the unique positive stationary solution $\left(u_{1}^{*}, u_{2}^{*}\right)$, which is globally exponentially stabilized under impulse control with the convergence rate $\frac{\lambda}{2}=0.75$.

Table 2.Comparisons the influences on the convergence rate $\frac{\lambda}{2}$ under different pulse frequency with the same other data

\begin{tabular}{|c|c|c|}
\hline & Example 4.1 & Example 4.3 \\
\hline Pulse amplitude & $\lambda_{\max } A_{k}=0.2$ & $\lambda_{\max } A_{k}=0.2$ \\
\hline Pulse intensity & same & same \\
\hline Pulse interval & $\left(t_{k+1}-t_{k}\right) \equiv 0.5$ & $\left(t_{k+1}-t_{k}\right) \equiv 0.3$ \\
\hline Pulse frequency & smaller & bigger \\
\hline Convergence rate & $\frac{\lambda}{2}=0.5$ & $\frac{\lambda}{2}=0.75$ \\
\hline
\end{tabular}

Remark 4. Table 2 reveals that under the same pulse amplitude, the higher the pulse frequency, the faster the convergence speed.

\section{Conclusions and further consideration}

The ecosystem with Dirichelt zero boundary value represents that the nature has limited resources, and population density of the species is zero on the edge of the limited ecological resources, which is entirely in line with some actual situations. Gilpin and Ayala in [5] pointed out that the model did not match a series of experimental data well. Via accurate data analysis, they proposed the nonlinear competition model with two-species, in which $\theta_{1}, \theta_{2}$ represent the nonlinear density restrictions. As pointed out in [6-8] that the nonlinear density restrictions model can match well the experimental data on drosophila melanogasters when $\theta_{i}$ was far less than 1 . So, in this paper, the author considers the nonlinear density restrictions model with $\theta_{i}<1$. Utilizing the fixed point theorem, variational method and Lyapunov function method results in the unique existence of the stationary solution of reaction-diffusion Gilpin-Ayala competition model, which is globally asymptotical input-to-state stability. Numerical examples illustrate that improving pulse frequency and pulse amplitude is helpful to make the ecosystem stabilized quickly. 
Now, the further consideration is, how to study the bi-stabilization of reaction-diffusion two species competition model with Dirichlet boundary value under invasion of infectious diseases. Especially in the novel coronavirus pneumonia epidemic today, it is an interesting problem.

Funding: The work is supported by the Application basic research project of science and Technology Department of Sichuan Province (No. 2020YJ0434) and the Major scientific research projects of Chengdu Normal University in 2019 (No. CS19ZDZ01)

Conflicts of Interest: The author declares no conflict of interest.

\section{References}

1. Rui Xu, M. A. J. Chaplain, F. A. Davidson. Travelling wave and convergence in stage-structured reaction-diffusion competitive models with nonlocal delays. Chaos, Solitons \& Fractals, 2006, 30(4), 974-992

2. Xiao-Xin Liao, Jia Li. Stability in gilpin-ayala competition models with diffusion. Nonlinear Analysis: Theory, Methods \& Applications, 1997, 28(10), 1751-1758.

3. Ling Bai, Ke Wang. Gilpin-Ayala model with spatial diffusion and its optimal harvesting policy. Applied Mathematics and Computation, 2005, 171(1), 531-546.

4. Yuanyuan Liu, Youshan Tao. Dynamics in a parabolic-elliptic two-species population competition model with cross-diffusion for one species. Journal of Mathematical Analysis and Applications, 2017, 456(11), 1-15.

5. Gilpin, M.E., Ayala, F.J., Global models of growth and competition. Proceedings of the National Academy of Sciences of the United States of America, 70 (1973) 3590-3593

6. Michael E. Gilpin, Francisco J. Ayala. Schoener's model and Drosophila competition. Theoretical Population Biology,1976 , 9(1), 12-14

7. William R. Thomas,Mark J. Pomerantz,Michael E. Gilpin. Chaos, Asymmetric Growth and Group Selection for Dynamical Stability. Ecology, 1980, 61,1312-1320. DOI: 10.2307/1939039.

8. Moore, Christopher M.,Catella, Samantha A.,Abbott, Karen C. Population dynamics of mutualism and intraspecific density dependence: How $\theta$-logistic density dependence affects mutualistic positive feedback. Ecological Modelling Volume 368, 24 January 2018, Pages 191-197

9. Qun Liu. Asymptotic properties of a stochastic n-species Gilpin-Ayala competitive model with Levy jumps and Markovian switching Communications in Nonlinear Science and Numerical Simulation, 2015, 26(1-3), $1-10$

10. Shuang Liu, Xinfeng Liu. Numerical Methods for a Two-Species Competition-Diffusion Model with Free Boundaries. Mathematics, 2018, 6(5), 72.

11. Ruofeng Rao. Stability Analysis of Nontrivial Stationary Solution and Constant Equilibrium Point of Reaction-Diffusion Neural Networks with Time Delays under Dirichlet Zero Boundary Value. Preprints 2020, 2020040277 (doi: 10.20944/preprints202004.0277.v4).

12. Fengde Chen.Some new results on the permanence and extinction of nonautonomous Gilpin-Ayala type competition model with delays. Nonlinear Analysis: Real World Applications, 2006, 7(5), 1205-1222

13. Fengde Chen. Permanence of a delayed non-autonomous Gilpin-Ayala competition model. Applied Mathematics and Computation, 2006, 179(1), 55-66

14. Manickam Iswarya ,Ramachandran Raja ,Grienggrai Rajchakit ,Jinde Cao ,Jehad Alzabut and Chuangxia Huang. Existence, Uniqueness and Exponential Stability of Periodic Solution for Discrete-Time Delayed BAM Neural Networks Based on Coincidence Degree Theory and Graph Theoretic Method. Mathematics, 2019, 7(11), 1055.

15. Ruofeng Rao and Shouming Zhong. Impulsive control on delayed feedback chaotic financial system with Markovian jumping. Advances in Difference Equations, 2020, 2020:50.

16. Yingkang Xie ,Zhen Wang and Bo Meng. Stability and Bifurcation of a Delayed Time-Fractional Order Business Cycle Model with a General Liquidity Preference Function and Investment Function. Mathematics, 2019, 7(9), 846;

17. Ruofeng Rao. Global Stability of a Markovian Jumping Chaotic Financial System with Partially Unknown Transition Rates under Impulsive Control Involved in the Positive Interest Rate. Mathematics, 2019, 7(7), 579 . 
18. Wenjuan Xie, Quanxin Zhu. Input-to-state stability of stochastic nonlinear fuzzy Cohen-Grossberg neural networks with the event-triggered control. International Journal of Control, 2020, 93(9):2043-2052.

19. Xiaodi Li, Xiaoli Zhang, Shiji Song. Effect of delayed impulses on input-to-state stability of nonlinear systems, Automatica, 2017, 76, 378-382

20. Xiaodi Li, Peng Li, Qing-guo Wang. Input/output-to-state stability of impulsive switched systems Systems \& Control Letters, 116, 2018, 1-7.

21. Peng Li, Xiaodi Li. Input-to-state stability of nonlinear impulsive systems via Lyapunov method involving indefinite derivative Mathematics and Computers in Simulation, 2019,155, 314-323

22. Haitao Zhu, Peng Li, Xiaodi Li, Haydar Akca. Input-to-state stability for impulsive switched systems with incommensurate impulsive switching signals. Communications in Nonlinear Science and Numerical Simulation, 2020, 80, 104969

23. Ruofeng Rao, Shouming Zhong, Xiongrui Wang. Stochastic stability criteria with LMI conditions for Markovian jumping impulsive BAM neural networks with mode-dependent time-varying delays and nonlinear reaction-diffusion. Communications in Nonlinear Science and Numerical Simulation, 2014, 19(1), 258-273 